\section{Handbook of Zoonoses: Identification and Prevention}

\section{Joann Colville and David L. Berryhill}

Mosby, St. Louis, Missouri, USA, 2007

\section{ISBN: 9780323044783}

Pages: 272; Price: US \$44.95

In "Handbook of Zoonoses: Identification and Prevention," authors Joann Colville and David L. Berryhill laud their book as an ideal reference for veterinarians, veterinary technicians, and professional students, and as a general resource for healthcare professionals to help them understand and manage zoonotic diseases. Information on common, and currently topical, zoonoses are included; the book addresses diseases caused by bacteria, viruses, parasites, fungi, and prions. For each disease, a common set of concepts are covered: the degree of illness and death associated with the disease; the etiology, hosts, and routes of transmission; a brief description of the disease manifestation in various animal species and in humans; general guidance for treatment in both animals and humans; and recommendations for prevention. Although the authors present some information on diseases that occur outside the United States, this handbook focuses more on diseases in the United States that readers may come into contact with or hear about on the news.

The terminology used to cover zoonotic diseases may be useful to healthcare professionals because basic information is provided in easily understandable language for lay patients and clients. As an actual source of information for healthcare professionals, however, this handbook is not as useful as other available texts. The book covers aspects of many of these diseases somewhat superficially and makes generalizations that could be misleading when examined in greater depth. This weakness is exacerbated by the fact that no references are provided, either as source data for citation or as pointers for readers looking for more information on a particular disease. Given the lack of overall detail in the handbook, this is a significant shortcoming. And, while an overall goal of the book is to provide information to help healthcare professionals manage zoonotic diseases, the authors do not present specific treatment guidance or common differential diagnoses for the diseases covered. Instead, the authors provide the occasional piece of trivia about certain diseases covered in the handbook that a lay reader may find engaging - that Ted Nugent wrote a song called "Cat Scratch Fever" and that rumors exist of a "Hollywood Tapeworm Diet." Overall, this handbook may be best suited for the lay person who has an interest in zoonotic diseases and some preexisting knowledge of disease transmission and pathogenesis.

\section{Kathleen Glynn*}

*Centers for Disease Control and Prevention, Atlanta, Georgia, USA

Address for correspondence: M. Kathleen Glynn, Bacterial Zoonoses Branch, Centers for Disease Control and Prevention, 1600 Clifton Rd, Mailstop C09, Atlanta, GA 30333, USA; email: mjg6@cdc.gov

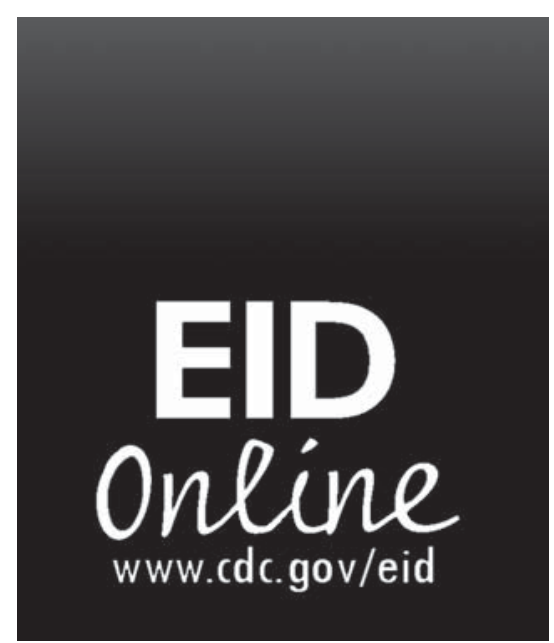

\section{The Microbiology Bench Companion}

\section{J. Michael Miller}

ASM Press, Washington, DC, USA, 2007

ISBN-10: 1555814026, ISBN-13:

978-1555814021

Pages: 120; Price: US \$36.90

Diagnostic testing for infectious diseases is an increasingly complex area of laboratory medicine. The microbial community continues to evolve and adapt to changing environmental influences, and the distribution of human pathogens has become more global. Our recognition of the spectrum of microorganisms that cause invasive human disease has exploded with the use of culture-independent methods to detect and characterize pathogens. Clinicians, epidemiologists, and public health officials can benefit from consultative interactions with laboratory professionals to assist with optimizing diagnostic test options and interpretation of test results. For technologists without access to boardcertified laboratory professionals who can guide the work-up of infectious agents in a microbiology laboratory, a concise guide can be extremely beneficial.

In this handbook, J. Michael Miller, a highly experienced clinical microbiologist, distills a great deal of information into 120 pages, largely formatted as tables and flowcharts. Molecular methods for detecting or identifying microorganisms are notably absent, which may reflect the author's intent to address readers who perform conventional diagnostic tests only. The handbook is divided into 3 sections. Section 1 focuses on routine laboratory bench algorithms for identifying bacteria, fungi, and parasites. Although most of this information can be found in clinical microbiology textbooks, the flowcharts are an easy reference, especially for medical technologists. Section 2 is entirely formatted 